\title{
FIXED POINTS OF MAPS OF $\beta N$
}

\author{
BY ZDENĚK FROLÍK
}

Communicated by R. D. Anderson, August 16, 1967

In this note all spaces are assumed to be uniformizable, and if $P$ is a space then $\beta P$ denotes a Stone-Cech compactification of $P$. If $P$ is a discrete space we shall think of the points of $P^{*}=\beta P-P$ as the free ultrafilters on $P$. The letter $N$ denotes the discrete space of natural numbers. The main results, Theorems $\mathrm{A}$ and $\mathrm{C}$, read as follows:

THEOREM A. If $M$ is a discrete space, $f$ is a homeomorphism of $\beta M$ into itself, and if $P_{k}$ is the set of all $k$-periodic points of $f$, then

$$
P_{k}=\operatorname{cl}\left(P_{k} \cap M\right) \text {. }
$$

In particular

TheOREM B. If $M$ is discrete, then no homeomorphism of $\beta M$ into $M^{*}$ has a fixed point.

Theorem C. If $M$ is a discrete space, and if $x$ is a fixed point of a continuous mapping $f$ of $\beta M$ into itself, then each neighborhood of $x$ contains an $f$-invariant neighborhood $V$ of $x$ (that means, with $f[V] \subset V)$. In addition, $V$ can be chosen of the form $\mathrm{cl} X, X \subset M$.

REMARK 1. For $M=N$ Theorem B reads: no type is a relative type of itself, i.e. $\langle t, t\rangle \in \Phi$ for no type $t$ (for terminology see [1]). Using the continuum hypothesis W. Rudin proved in [5] that $N^{*}$ is not homogeneous. In [1] the present author proved the nonhomogeneity of $N^{*}$ without using the continuum hypothesis. Theorem B gives another, very simple, proof: if $h$ is a homeomorphism of $N^{*}$ onto itself and $h x=y$, then evidently the sets of all relative types of $x$ and $y$ coincide. Thence, by Theorem $\mathrm{B}$, if the type of a point $x$ is a relative type of $y$, then $h x=y$ for no homeomorphism $h$ of $N^{*}$. Note that this proof establishes the existence of $\exp \exp \boldsymbol{\aleph}_{0}$ of orbits of the homeomorphism group of $N^{*}$.

REMARK 2. Theorem $\mathrm{B}$ in the particular case $M=N$ was announced in [2]. M. Katètov reproved that result in [3] (his proof works for any $M$ with $f[M]$ an $r$-set, see Example 2 below). The original proof, which works in the countable case only, is also given below because it is of some interest in itself.

REMARK 3. It would be interesting to find an analogue of Theorem $\mathrm{B}$ for any continuous mapping of $\beta M$. The following examples suggest that the entire orbit of $M$ must be considered. 
ExAmple 1. There exists a continuous $f: \beta N \rightarrow N^{*}$ such that:

(a) $f: N \rightarrow f[N]$ is one-to-one,

(b) $f[N]$ is a countable union of discrete sets, and

(c) $f^{2}[N]$ is a singleton $(x)$. (Thence, $x$ is a unique fixed point of $f$.)

To exhibit such an $f$, choose discrete countable infinite sets $Y_{n} \subset N^{*}$, $n \geqq 1$, with $Y_{n+1} \subset \mathrm{cl} Y_{n}-Y_{n}$, and a point $y$ in the intersection of all cl $Y_{n}$. Next choose a decomposition $\left\{N_{n}\right\}$ of $N$ into infinite sets, and then select $x_{n}$ in $N_{n}^{*}, n \geqq 1$, such that the relative type of $x_{n}$ coincides with the type of $y$ with respect to $Y_{n}$. Let $X_{0}$ be the set of all $x_{n}$, and choose a homeomorphism $g$ of $\beta N$ onto cl $X_{0}$. Put $x=g y, X_{n}=g\left[Y_{n}\right]$. Now we can choose a one-to-one mapping $F$ of $N$ onto the union $X$ of all $X_{n}$ such that $N_{n}$ is mapped onto $X_{n}$, and if $f$ is the Cech-Stone extension of $F$ then $f x_{n}=x$. Thus $f\left[X_{0}\right]=(x)$, and hence $f^{2}[N]=f[X]$ $=(x)$ by continuity of $f$.

ExAmple 2. If $f: \beta M \rightarrow M^{*}$ is continuous, and $M$ is discrete then the following statements are equivalent: (a) $f$ is a retraction, (b) $f^{2} m=f m$ for all $m$ in $M$, and (c) $f m \in \operatorname{cl} f^{-1}[\mathrm{fm}]$ for all $m$ in $M$. The sets of the form $f[M]$ with $f$ a retraction are called $r$-sets.

EXAmple 3. There exists a continuous $f: \beta N \rightarrow N^{*}$ such that $f[N]$ is discrete, and $f^{2}[N]$ is a singleton disjoint to $f[N]$. Take a type $t$ and a decomposition $\left\{N_{n}\right\}$ of $N$ with $N_{n}$ infinite, pick $x_{n}$ in $N_{n}^{*}$ of type $t$, and select any point $x$ of the type $t$ with respect to the set $X$ of all $x_{n}$. Finally, take $f$ such that the restriction to any $\mathrm{cl} N_{n}$ is a homeomorphism onto $\mathrm{cl} X$ with $f x_{n}=x$.

Let Theorem $A^{\prime}$ be the proposition obtained from Theorem A by replacing "into" by "onto." In the particular case $M=N$ Theorem A' is essentially due to R. Raimi [4]. Theorems $A^{\prime}$ and $B$ are particular cases of Theorem $A$. On the other hand Theorem $A$ is derived from $A^{\prime}$ and $B$ as follows:

It is enough to consider the case $k=1$. It follows from $B$ that if $x$ is a fixed point of a homeomorphism $f$ of $\beta M$ into itself then $x \in \mathrm{cl}(M \cap f[M])$. Then an application of $A^{\prime}$ to an appropriate homeomorphism $h$ of $\beta M$ onto itself gives the desired result ( $h m=f m$ for $m$ in $M \cap f[M])$.

For a proof of $\mathrm{A}^{\prime}$ we refer to [3] or [2] (in the particular case $M=N)$. Now $\mathrm{C}$ will be proved, then $\mathrm{B}$ will be derived from $\mathrm{C}$, and finally a simple proof of a generalization of $B$ in the particular case $M=N$ will be given. In conclusion an extension of [1] to any cardinal will be sketched.

Proof of Theorem C. Let $M$ be an infinite discrete space, $f$ be a continuous mapping of $\beta N$ into itself, and let $f x=x$. If $x \in M$, then the statement is trivial. Assume $x \notin M$. Given a neighborhood $U$ of $x$, 
choose an $X \subset M$ such that $\mathrm{cl} X$ is neighborhood of $x$ (i.e., $x \in \mathrm{cl} X$ ) and cl $X \subset U$. We shall exhibit a $Y \subset X$ with $f[Y] \subset \operatorname{cl} Y, x \in \mathrm{cl} Y$. Define by induction:

$$
\begin{aligned}
X_{1} & =E\{y \mid y \in X, f y \notin \operatorname{cl} X\}, \\
P_{\alpha} & =\bigcup\left\{X_{\beta} \mid \beta<\alpha, \beta \text { isolated }\right\}, Q_{\alpha}=U\left\{X_{\beta} \mid \beta<\alpha\right\}, \\
X_{\alpha} & =E\left\{y \mid y \in X-Q_{\alpha}, f y \in \operatorname{cl} Q_{\alpha}\right\} \text { if } \alpha \text { is isolated, } \\
& =E\left\{y \mid y \in X-Q_{\alpha}, f y \in \operatorname{cl} P_{\alpha}\right\} \text { if } \alpha \text { is limit ordinal. }
\end{aligned}
$$

Consider the sets:

$Z_{\text {limit }}=\bigcup\left\{X_{\beta} \mid \beta\right.$ is a limit ordinal $\}, Z_{\text {odd }}=\bigcup\left\{X_{\beta} \mid \beta\right.$ is odd $\}$, $Z_{\text {even }}=\bigcup\left\{X_{\beta} \mid \beta\right.$ is even and isolated $\}, Y=X-\left(Z_{\text {limit }} \cup Z_{\text {odd }} \cup Z_{\text {even }}\right)$.

First, let us prove that $f[Y] \subset \mathrm{cl} Y$. There exists an isolated $\alpha \geqq 0$ with $X_{\alpha}=\varnothing$ because $\left\{X_{\beta}\right\}$ is a disjoint family and, if $X_{\beta}=\varnothing$ for some isolated $\beta$, then $X_{\lambda}=\varnothing$ for all $\lambda \geqq \beta$. By definition of $X_{\alpha}$ (and $X_{1}$ ) $E\left\{y \mid y \in X-Q_{\alpha}, f y \notin \operatorname{cl}\left(X-Q_{\alpha}\right)\right\}=\left(X_{\alpha} \cup\left(X-Q_{\alpha}\right) \cap X_{1}\right)=\varnothing$.

Thus $Y=X-Q_{\alpha}$, and hence $F[Y] \subset \mathrm{Cl} Y$. It remains to show that cl $Y$ is a neighborhood of $x$, i.e. $x \in \mathrm{cl} Y$. It suffices to show that the closure of none of the $Z$ 's is a neighborhood of $x$. If $W \subset M$ and $\mathrm{cl} W$ is a neighborhood of $x$, then necessarily the closure of the set

$$
\rho W=E\{y \mid y \in W, f y \in \operatorname{cl} W\}
$$

is also a neighborhood of $x$ because of the continuity of $f$ and $f x=x$. So, it is enough to show that $\rho Z_{\text {odd }}=\rho Z_{\text {even }}=\rho Z_{\text {limit }}=\varnothing$, and this follows easily from the following relations and the fact that the sets $N-X, Z_{\text {odd }}, Z_{\text {even }}$ and $Z_{\text {limit }}$ are disjoint:

$$
\begin{aligned}
& f\left[Z_{\text {limit }}\right] \subset \operatorname{cl}\left[Z_{\text {odd }} \cup Z_{\text {even }}\right], \quad f\left[Z_{\text {even }}\right] \subset \operatorname{cl} Z_{\text {odd }}, \\
& \left.f\left[Z_{\text {odd }}\right] \subset \operatorname{cl}(N-X) \cup Z_{\text {even }} \cup Z_{\text {limit }}\right) .
\end{aligned}
$$

The verification is routine.

Proof of Theorem B. Assume that $x$ is a fixed point of a homeomorphism $f: \beta M \rightarrow M^{*}$. Consider the collection Tl of all subsets $X$ of $M$ which admits a disjoint decomposition $X=X_{1} \cup X_{2} \cup X_{3}$, such that $f\left[X_{i}\right] \subset \operatorname{cl}\left(X_{j} \cup X_{k}\right)$ for $i \neq j \neq k$. Clearly, the union of any disjoint subcollection of $\mathfrak{T}$ belongs to $\mathfrak{T}$, and $x$ belongs to the closure of no $X$ in $\mathfrak{T}$. Let $X$ be the union of a maximal disjoint subcollection $\mathfrak{X}$ of $\mathfrak{T}$. We shall exhibit a nonvoid $Y \in \mathscr{T}($ disjoint to $X$; this will contradict the maximality of $\mathcal{X}$ and conclude the proof. Since $\operatorname{cl}(M-X)$ is a neighborhood of $x$, by Theorem $\mathrm{C}$ there exists a $V \subset M-X$ such that 
cl $V$ is an $f$-invariant neighborhood of $x$. Choose any infinite subset $Y_{3}$ of $V$ such that $f\left[Y_{3}\right] \cap c l Y_{3}=\varnothing$. Pick $Z_{1} \subset V-Y_{3}$ such that $\operatorname{cl} Z_{1} \cap f[M]=f\left[Y_{3}\right]$, and by induction $Z_{n+1} \subset V-\left(Y_{3} \cup \cup\left\{Z_{k} \mid k \leqq n\right\}\right.$, $n \in N$, such that $\operatorname{cl} Z_{1} \cap f[M]=f\left[Y_{3}\right]$,

$$
f\left[Z_{n}\right]-\operatorname{cl} Y_{3}=f[M] \cap \operatorname{cl} Z_{n+1} .
$$

To justify this, we need the facts that $f[M]$ is normally embedded in $\beta M$ (and this follows from the fact that $\mathrm{cl} f[M]$ is a Stone-Cech compactification of $f[M])$, and $f$ is one-to-one on $M$. If $Y_{1}$ is the union of $Z_{n}$ with $n$ odd, and if $Y_{2}$ is the union of all the other $Z_{n}$ 's, then $f\left[Y_{3}\right]$ $\subset \mathrm{cl} Z_{1} \subset \mathrm{cl} Y_{1}$, and $f\left[Y_{i}\right] \subset \operatorname{cl}\left(Y_{j} \cup Y_{3}\right)$ for $i \neq j, i, j=1,2$. Consequently, the set $Y=Y_{1} \cup Y_{2} \cup Y_{3}$ belongs to $\Re$, and clearly $\varnothing \neq Y$ $\subset M-X$.

For $M=N$ Theorem $\mathrm{B}$ is an immediate consequence of the following

Proposition. If $f$ is a continuous mapping of $\beta N$ into itself such that

$$
\operatorname{cl} f^{n+1}[N] \cap f^{n}[N]=\varnothing
$$

for all $n$, then $f$ has no fixed point.

Proof. Put $N_{n}=f^{n}[N]$. If $X \subset \cup\left\{N_{n}\right\}$, and $X \cap N_{n}$ is finite for all $n$, then $X$ is discrete. Choose sets $X$ and $Y$ such that $X \cap Y=\varnothing$, the orbit of each $n \in N$ intersects both $X$ and $Y$, and the sets $X \cap N_{n}$, $Y \cap N_{n}$ are finite for all $n$ (if $f$ is a homeomorphism, we can take $E\left\{f_{n}^{n}\right\}$ for $X$ and $E\left\{f_{n}^{n+1}\right\}$ for $Y$ ). By Theorem C, $x$ belongs to the closure of $X$ as well as that of $Y$, and this contradicts the fact that $X \cup Y$ is discrete.

REMARK 4. It is clear that the theory of types of ultrafilters on countable sets developed in [1] carries over to any ultrafilters if we restrict ourselves to the relative types with respect to $r$-sets (cf. Example 2). Every $r$-set is a discrete normally embedded set, and I do not know whether the converse is true or not. However, Theorem $B$ says that the type of any point is distinct from all relative types, even from those with respect to the normally embedded discrete sets.

REMARK 5. Theorem A can be extended to compact extremally disconnected spaces (the statement is modified as follows: the set of all $k$-periodic points is both closed and open). Theorem $\mathrm{C}$ extends to any extremally disconnected space. The details will appear elsewhere in connection with homogeneity problems.

\section{REFERENCES}

1. Z. Frolik, Sums of ultrafilters, Bull. Amer. Math. Soc. 73 (1967), 87-91. 
2. - Types of ultrafilters, Proc. of the 2nd Topology Symposium 1966, Academia 1967, Praha.

3. M. Katětov, A theorem on mappings, Comment Math. Univ. Carolinae. 8 (1967), 431-433.

4. R. Raimi, Homeomorphisms and invariant measures for $\beta N-N$, Duke Math. J. 13 (1966), 1-12.

5. W. Rudin, Homogeneity problems in the theory of Cech compactification, Duke Math. J. 23 (1956), 409-420.

Louisiana State University, Baton Rouge, University of California, Riverside, and Mathematics Institute, Praha

\section{REPRESENTATION OF FRACTIONAL POWERS OF INFINITESIMAL GENERATORS OF SEMIGROUPS}

BY H. BERENS, P. L. BUTZER AND U. WESTPHAL ${ }^{1}$

Communicated by Felix Browder, September 1, 1967

1. Introduction. Let $X$ be a real or complex Banach space and $\mathbb{E}(X)$ the Banach algebra of endomorphisms of $X$. Let $\{T(u) ; u \geqq 0\}$ be an equibounded semigroup of operators of class $\left(\mathfrak{G}_{0}\right)$ in $\mathbb{E}(X)$ with infinitesimal generator $A . A$ is a closed linear operator with domain $D(A)$ dense in $X$. For these concepts see e.g. E. Hille-R. S. Phillips $[5$, Chapters X-XII].

One purpose of this note is to give a representation for the fractional power $(-A)^{\gamma}, \gamma>0$, of the operator $(-A)$. The result obtained will be a generalization of one due to J. L. Lions-J. Peetre [7, Chapter VII,\$2]: Let $\gamma$ be a positive integer. An element $f \in X$ belongs to $D\left((-A)^{\gamma}\right)$ if and only if the integral

$$
\frac{1}{C_{\gamma, r}} \int_{\epsilon}^{\infty} \frac{[I-T(u)]^{r f}}{u^{1+\gamma}} d u \quad(\epsilon>0 ; r \text { any integer }>\gamma) .
$$

converges in norm as $\epsilon \rightarrow 0_{+}$, the constant $C_{\gamma, r}$ being given by

$$
C_{\gamma, r}=\int_{0}^{\infty} \frac{\left(1-e^{-u}\right)^{r}}{u^{1+\gamma}} d u .
$$

The limit is then equal to $(-A)^{\gamma} f$.

1 The research of this author was supported by a DFG grant. 